\title{
Otras disposiciones de interés para la Comunidad Autónoma ${ }^{1}$
}

\section{PRESIDENGIA}

Ley 5/2017, de 5 de diciembre, del Presupuesto de la Comunidad Autónoma de Andalucía para el año 2018 (BOJA 239, de 15 de diciembre).

Ley 6/2017, de 27 de diciembre, por la que se modifica la Ley 3/2013, de 24 de julio, por la que se aprueba el Plan Estadístico y Cartográfico de Andalucía 20132017 (BOJA 248, de 29 de diciembre).

\section{CONSEJERÍA DE LA PRESIDENGIA, ADMINISTRAGIÓN LOGAL Y MEMORIA DEMOGRÁTICA}

Decreto 142/2017, de 29 de agosto, por el que se modifican los Decretos 204/2015, de 14 de julio, por el que se establece la estructura orgánica de la Consejería de la Presidencia y Administración Local, 209/2015, de 14 de julio, por el que se establece la estructura orgánica de la Consejería de Igualdad y Políticas Sociales, 213/2015, de 14 de julio, por el que se establece la estructura orgánica de la Consejería de Cultura, y 214/2015, de 14 de julio, por el que se establece la estructura orgánica de la Consejería de Justicia e Interior (BOJA 169, de 4 de septiembre).

Acuerdo de 26 de septiembre de 2017, del Consejo de Gobierno, por el que se aprueba la formulación del Plan de Acción de Gobierno Abierto de la Junta de Andalucía (BOJA 189, de 2 de octubre).

Orden de 19 de octubre de 2017, por la que se acuerdan actuaciones específicas en Memoria Democrática en determinados municipios andaluces (BOJA 203, de 23 de octubre).

Decreto 172/2017, de 24 de octubre, por el que se aprueba el Plan Director de las Dehesas de Andalucía, se crea su Comité de Seguimiento y se modifica el Decreto 57/2011, de 15 de marzo, por el que se regula la Comisión Andaluza para la Dehesa y el Decreto 530/2004, de 16 de noviembre, por el que se regula la composición, las funciones y el régimen de funcionamiento del Consejo Andaluz de Biodiversidad (BOJA 207, de 27 de octubre).

Subsección preparada por MARÍA DEL CARMEN NÚÑEZ LOZANO, Catedrática de Derecho Administrativo, Centro de Investigación en Patrimonio Histórico, Cultural y Natural, Universidad de Huelva. 
Acuerdo de 14 de noviembre de 2017, del Consejo de Gobierno, por el que se modifican los Anexos I y III del Acuerdo del Consejo de Gobierno de 21 de junio de 2005 por el que se determinan los servicios y actividades de carácter sanitario susceptibles de ser retribuidos mediante precios públicos, en los supuestos en los que exista un tercero obligado al pago de la asistencia (BOJA 221, de 17 de noviembre).

\section{GONSEJERÍA DE EGONOMÍA Y GONOGIMIENTO}

Decreto 151/2017, de 26 de septiembre, por el que se aprueba el Programa Estadístico y Cartográfico de la Comunidad Autónoma de Andalucía para el año 2017, y se modifica el Decreto 372/2009, de 17 de noviembre, por el que se regula la organización y funcionamiento del Sistema Estadístico de Andalucía (BOJA 189, de 2 de octubre).

Decreto 152/2017, de 26 de septiembre, por el que se establecen y regulan los Órganos Colegiados del Plan Estratégico de Internacionalización de la Economía Andaluza Horizonte 2020 (BOJA 189, de 2 de octubre).

Resolución de 8 de noviembre de 2017, de la Dirección General de Universidades, por la que se hace público el Acuerdo de 30 de octubre de 2017, de la Comisión Coordinadora Interuniversitaria de Andalucía, por la que se actualizan los plazos y los calendarios para la participación en el procedimiento para la obtención de los requisitos que permitan el acceso a los estudios universitarios de grado de las personas mayores de 40 y 45 años, que no posean otros requisitos para el acceso a la Universidad, que se celebre en el curso 2017-2018 (BOJA 226, de 24 de noviembre).

Resolución de 8 de noviembre de 2017, de la Dirección General de Universidades, por la que se hace público el Acuerdo de 30 de octubre de 2017, de la Comisión Coordinadora Interuniversitaria de Andalucía por el que se actualizan los plazos y el calendario para la participación en la Prueba de Acceso a la Universidad para mayores de 25 años que se celebre en el curso 2017-2018 (BOJA 226, de 24 de noviembre).

Resolución de 4 de diciembre de 2017, de la Dirección General de Universidades, por la que se establece el número de profesorado emérito de las Universidades Públicas de Andalucía, para el curso académico 2017/2018 (BOJA 239, de 15 de diciembre).

Decreto 197/2017, de 12 de diciembre, por el que se aprueba la modificación de los Estatutos de la Universidad de Cádiz, aprobados por Decreto 281/2003, de 7 de octubre (BOJA 239, de 15 de diciembre).

Resolución de 12 de diciembre de 2017, de la Dirección General de Universidades, por la que se hace público el Acuerdo de 29 de noviembre de 2017 de la Comisión del Distrito Único Universitario de Andalucía, por el que se establece el 
procedimiento para el ingreso en los másteres universitarios que se impartan en el curso 2018-2019 (BOJA 242, de 20 de diciembre).

\section{GONSEJERÍA DE HAGIENDA Y ADMINISTRAGIÓN PÚBLIGA}

Orden de 18 de septiembre de 2017, por la que se establece la distribución de los créditos derivada del Decreto de la Presidenta 12/2017, de 8 de junio, de la Vicepresidencia y sobre reestructuración de Consejerías, así como las reglas aplicables a la modificación, gestión y fiscalización de los créditos afectados (BOJA 184, de 25 de septiembre).

Resolución de 19 de septiembre de 2017, de la Dirección General de Patrimonio, por la que se deroga la declaración de necesaria uniformidad de microordenadores y periféricos, cuyo acuerdo marco de homologación ha expirado su vigencia (BOJA 190, de 3 de octubre).

Decreto 165/2017, de 17 de octubre, por el que se autoriza la concertación de operaciones de endeudamiento hasta un importe máximo de mil quinientos diez millones cincuenta mil euros (BOJA 203, de 23 de octubre).

Resolución de 19 de octubre de 2017, de la Dirección General de Recursos Humanos y Función Pública, por la que se determina la cuantía de la ayuda de acción social para atención a personas con discapacidad para el ejercicio 2017 (BOJA 213, de 7 de noviembre).

Resolución de 20 de octubre de 2017, de la Dirección General de Patrimonio, por la que se aprueba el Plan de Inspección del Juego y Apuestas, para el período 2018-2020 (BOJA 223, de 21 de noviembre).

Orden de 6 de noviembre de 2017, sobre cierre del ejercicio presupuestario de 2017 (BOJA 215, de 9 de noviembre).

Orden de 24 de noviembre de 2017, por la que se establecen normas para la gestión, seguimiento y control de los ingresos y gastos de los servicios Transferencias Finalistas y Otros ingresos finalistas (BOJA 231, de 1 de diciembre).

Resolución de 13 de diciembre de 2017, de la Dirección General de Tesorería y Deuda Pública, por la que se hace público el calendario de subastas ordinarias del Programa de Emisión de Pagarés de la Junta de Andalucía para el año 2018 y se convocan las correspondientes subastas a realizar dentro del mismo (BOJA 245, de 26 de diciembre).

Orden de 15 de diciembre de 2017, por la que se publica la modificación del Anexo I y los valores de las variables establecidas en el artículo 10 de la Ley 6/2010, 
de 11 de junio, reguladora de la Participación de las Entidades Locales en los tributos de la Comunidad Autónoma de Andalucía (BOJA 243, de 21 de diciembre).

Acuerdo de 26 de diciembre de 2017, del Consejo de Gobierno, por el que se aprueba el Acuerdo de la Mesa General de Negociación Común del Personal Funcionario, Estatutario y Laboral de la Administración de la Junta de Andalucía, de 18 de diciembre de 2017, sobre medidas transitorias para ordenar la jornada de trabajo y horarios del personal empleado público de la Junta de Andalucía (BOJA 246, de 27 de diciembre).

\section{GONSEJERÍA DE EDUGACIÓN}

Resolución de 12 de septiembre de 2017, de la Dirección General de Profesorado y Gestión de Recursos Humanos, por la que se determina el desarrollo de las líneas estratégicas de formación del profesorado establecidas en el III Plan Andaluz de Formación Permanente del Profesorado y la elaboración de los proyectos de formación para el curso 2017/18 (BOJA 183, de 22 de septiembre).

Orden de 28 de septiembre de 2017, por la que se hace público el modelo de documento administrativo en el que se formalizarán los convenios con los Ayuntamientos, Diputaciones Provinciales y otras Entidades Locales, para el uso de las instalaciones de los centros docentes públicos fuera del horario escolar (BOJA 192, de 5 de octubre).

Orden de 17 de octubre de 2017, por la que se regula el procedimiento para obtener el reconocimiento como Instituto Histórico Educativo de Andalucía (BOJA 209, de 31 de octubre).

Orden de 10 de noviembre de 2017, por la que se desarrolla el procedimiento para la selección y el nombramiento de los directores y las directoras de los centros docentes públicos no universitarios de los que es titular la Junta de Andalucía (BOJA 222, de 20 de noviembre).

Decreto 183/2017, de 14 de noviembre, por el que se modifica el Decreto 207/2015, de 14 de julio, por el que se establece la estructura orgánica de la Consejería de Educación (BOJA 225, de 23 de noviembre).

Decreto 194/2017, de 5 de diciembre, por el que se aprueban los Estatutos de la Agencia Pública Andaluza de Educación (BOJA 236, de 12 de diciembre).

Resolución de 11 de diciembre de 2017, de la Dirección General de Planificación y Centros, por la que se establece el calendario de actuaciones del procedimiento para solicitar acogerse al régimen de conciertos educativos, o la renovación 
o modificación de los mismos, para el curso académico 2018/19 (BOJA 245, de 26 de diciembre).

Orden de 28 de diciembre de 2017, por la que se establece la ordenación y el currículo de la Educación Secundaria Obligatoria para personas adultas en la Comunidad Autónoma de Andalucía (BOJA 249, de 30 de diciembre).

\section{CONSEJERÍA DE SALUD}

Resolución de 22 de septiembre de 2017, de la Dirección General de Profesionales del Servicio Andaluz de Salud, por la que se dispone la aprobación y publicación del texto refundido y actualizaciones del Pacto de Mesa Sectorial de Sanidad, suscrito entre la Administración Sanitaria de Andalucía-SAS y las Organizaciones Sindicales que se citan, el 26 de junio de 2017, sobre sistema de selección de personal estatutario temporal para puestos básicos en el Servicio Andaluz de Salud (BOJA 192, de 5 de octubre).

\section{CONSEJERÍA DE IGUALDAD Y POLÍTIGAS SOGIALES}

Acuerdo de 17 de octubre de 2017, del Consejo de Gobierno, por el que se aprueba el IV Plan Andaluz del Voluntariado para el período 2017-2020 (BOJA 205, de 25 de octubre).

Decreto 175/2017, de 31 de octubre, por el que se regula el contenido y procedimiento de elaboración del Informe Bienal de la Ley 2/2014, de 8 de julio, integral para la no discriminación por motivos de identidad de género y reconocimiento de los derechos de las personas transexuales de Andalucía (BOJA 213, de 7 de noviembre).

Acuerdo de 31 de octubre de 2017, del Consejo de Gobierno, por el que se aprueba el Plan Integral para la inclusión de la Comunidad Gitana de Andalucía para el período 2017-2020 (BOJA 213, de 7 de noviembre).

Orden de 28 de noviembre de 2017, por la que se modifica la Orden de 10 de agosto de 2017, por la que se establece la distribución de las cantidades a percibir por las entidades locales para la financiación de ayudas económicas familiares correspondientes al ejercicio 2017 (BOJA 232, de 4 de diciembre).

Orden de 4 de diciembre de 2017, por la que se determina la composición y funcionamiento de la Comisión Estadística y Cartográfica y de la Unidad Estadística y Cartográfica de la Consejería de Igualdad y Políticas Sociales (BOJA 236, de 12 de diciembre). 
Orden de 5 de diciembre de 2017, por la que se modifica la Orden de 10 de octubre de 2013, por la que se regulan las Ayudas Económicas Familiares y su gestión mediante la cooperación entre la Junta de Andalucía y las Entidades Locales (BOJA 239, de 15 de diciembre).

Decreto-ley 3/2017, de 19 de diciembre, por el que se regula la Renta Mínima de Inserción Social en Andalucía (BOJA 245, de 26 de diciembre).

Acuerdo de 19 de diciembre de 2017, del Consejo de Gobierno, por el que se establecen ayudas sociales de carácter extraordinario a favor de las personas perceptoras de las pensiones del Fondo de Asistencia Social y de las beneficiarias del Subsidio de Garantía de Ingresos Mínimos para el año 2018 (BOJA 244, de 22 de diciembre).

Acuerdo de 19 de diciembre de 2017, del Consejo de Gobierno, por el que se establecen ayudas sociales de carácter extraordinario a favor de pensionistas por jubilación e invalidez en sus modalidades no contributivas, para el año 2018 (BOJA 244, de 22 de diciembre).

Acuerdo de 19 de diciembre de 2017, del Consejo de Gobierno, por el que se distribuyen créditos entre Ayuntamientos de municipios con población superior a 20.000 habitantes y Diputaciones Provinciales, al objeto de financiar el refuerzo de los servicios sociales comunitarios en el desarrollo de las competencias atribuidas en materia de dependencia (BOJA 244, de 22 de diciembre).

Corrección de errores de la Orden de 26 de julio de 2017, por la que se modifica la Orden de 11 de febrero de 2004, por la que se regulan las prestaciones económicas a las familias acogedoras de menores (BOJA 236, de 12 de diciembre).

\section{CONSEJERÍA DE EMPLEO, EMPRESA Y COMERGIO}

Orden de 25 de julio de 2017, por la que se regula la tarjeta de acreditación de la identidad de las personas que ejercen la inspección en materia de comercio en el ámbito de la Junta de Andalucía (BOJA 179, de 18 de septiembre).

Acuerdo de 5 de septiembre de 2017, del Consejo de Gobierno, por el que se aprueba la formulación del Plan Estratégico para la incorporación de la ciudadanía a la Sociedad y la Economía Digital (BOJA 179, de 18 de septiembre; corrección de errores en el BOJA 211, de 3 de noviembre).

Orden de 5 de septiembre de 2017, por la que se aprueba la modificación de la Estrategia de Infraestructuras de Telecomunicaciones de Andalucía 2020 (BOJA 179, de 18 de septiembre).

Orden de 15 de septiembre de 2017, por la que se exceptúa a las entidades que se relacionan en el Anexo adjunto de las limitaciones establecidas en el artículo 124.1 
del Texto Refundido de la Ley General de Hacienda Pública de la Junta de Andalucía, aprobado por Decreto Legislativo 1/2010, de 2 de marzo (BOJA 191, de 4 de octubre).

Orden de 29 de septiembre de 2017, por la que se modifica la Orden de 2 de junio de 2016, por la que se regulan los programas de Escuelas Taller, Casas de Oficios, Talleres de Empleo y Unidades de Promoción y Desarrollo en la Junta de Andalucía, y se establecen las bases reguladoras de la concesión de ayudas públicas en régimen de concurrencia competitiva a dichos programas (BOJA 191, de 4 de octubre).

Resolución de 30 de septiembre de 2017, de la Dirección General de Telecomunicaciones y Sociedad de la Información, por la que se asignan las funciones de la Unidad de Seguridad TIC Corporativa (BOJA 193, de 6 de octubre).

Orden de 9 de octubre de 2017, por la que se establece el calendario de domingos y festivos en que los establecimientos comerciales podrán permanecer abiertos al público durante el año 2018 (BOJA 198, de 16 de octubre).

Resolución de 9 de noviembre de 2017, de la Dirección General de Industria, Energía y Minas, por la que se modifica el Anexo II de la Orden de 5 de marzo de 2013, por la que se dictan normas de desarrollo del Decreto 59/2005, de 1 de marzo, por el que se regula el procedimiento para la instalación, ampliación, traslado y puesta en funcionamiento de los establecimientos industriales, así como el control, responsabilidad y régimen sancionador de los mismos (BOJA 225, de 23 de noviembre).

Acuerdo de 14 de noviembre de 2017, del Consejo de Gobierno, por el que se aprueba la Estrategia Andaluza de Seguridad y Salud en el Trabajo 2017-2022 (BOJA 222, de 20 de noviembre).

Resolución de 22 de noviembre de 2017, de la Dirección General de Industria, Energía y Minas, por la que se aprueban los Planes Generales de Inspección en el área de industria, energía y minas para el bienio 2018-19 (BOJA 231, de 1 de diciembre).

Resolución de 30 de noviembre de 2017, de la Dirección General de Comercio, por la que se autoriza a determinados municipios la permuta de un domingo o festivo del calendario en que los establecimientos comerciales podrán permanecer abiertos al público durante el año 2018 (BOJA 234, de 7 de diciembre).

Decreto 192/2017, de 5 de diciembre, por el que se aprueba el Programa de Fomento del Empleo Industrial y Medidas de Inserción Laboral en Andalucía (BOJA 238, de 14 de diciembre).

Resolución de 18 de diciembre de 2017, de la Dirección General de Relaciones Laborales y Seguridad y Salud Laboral, por la que se publica la relación de fiestas 
locales de los municipios de la Comunidad Autónoma de Andalucía para el año 2018 (BOJA 244, de 22 de diciembre).

Resolución de 19 de diciembre de 2017, de la Dirección General de Comercio, por la que se aprueba el Plan de Inspección Comercial de Andalucía para el año 2018 (BOJA 246, de 27 de diciembre).

Orden de 19 de diciembre de 2017, por la que se otorga el reconocimiento de la condición de oficial a las Ferias Comerciales de Andalucía y se aprueba el calendario para el año 2018 (BOJA 247, de 28 de diciembre).

Orden de 26 de diciembre de 2017, por la que se aprueba el Plan Estratégico de Subvenciones del Instituto Andaluz de Prevención de Riesgos Laborales para el período 2017-2020 (BOJA 248, de 29 de diciembre).

Orden de 26 de diciembre de 2017, por la que se prorrogan los efectos de la Orden de 23 de enero de 2015, por la que se aprueba el Plan de Activación del Comercio Ambulante en Andalucía para el periodo 2015/2017 (BOJA 248, de 29 de diciembre).

\section{CONSEJERÍA DE FOMENTO Y VIVIENDA}

Orden de 18 de diciembre de 2017, de desarrollo y tramitación del Programa de Regeneración del Espacio Público Urbano en la Comunidad Autónoma de Andalucía (BOJA 243, de 21 de diciembre).

\section{GONSEJERÍA DE TURISMO Y DEPORTE}

Acuerdo de 19 de septiembre de 2017, del Consejo de Gobierno, por el que se declara municipio turístico al municipio de Punta Umbría (Huelva) (BOJA 184, de 25 de septiembre).

\section{GONSEJERÍA DE GULTURA}

Decreto 156/2017, de 3 de octubre, por el que se crea el Museo Ibero (BOJA 193, de 9 de octubre).

Orden de 6 de noviembre de 2017, por la que se determina la composición y funcionamiento de la Comisión Estadística y Cartográfica y de la Unidad Estadística y Cartográfica de la Consejería de Cultura (BOJA 217, de 13 de noviembre; corrección de errata en el BOJA 219, de 15 de noviembre).

\section{GONSEJERÍA DE JUSTIGIA E INTERIOR}

Resolución de 29 de noviembre de 2017, de la Secretaría General para la Justicia, por la que se publica el convenio entre el Ministerio de Justicia y la Comunidad 
Autónoma de Andalucía, por el que se formalizan los compromisos financieros del Acuerdo de Consejo de Ministros de 25 de agosto de 2017 en el que se aprueba la distribución del crédito del programa para reforma de la Administración de Justicia (BOJA 237, de 13 de diciembre).

\section{CONSEJERÍA DE AGRIGULTURA, PESGA Y DESARROLLO RURAL}

Resolución de 14 de septiembre de 2017, de la Dirección General de Ayudas Directas y de Mercados, por la que se declara con carácter excepcional el uso del pastoreo o siega con fines productivos en barbechos declarados como superficie de interés ecológico en el pago verde, para la campaña de solicitud 2017 (BOJA 183, de 22 de septiembre).

Resolución de 18 de septiembre de 2017, de la Dirección General de la Producción Agrícola y Ganadera, por la que se declara oficialmente la existencia de los organismos nocivos Epitrix papa y Epitrix cucumeris, se establecen nuevas zonas demarcadas, así como medidas fitosanitarias obligatorias para su control, en la Comunidad Autónoma de Andalucía (BOJA 183, de 22 de septiembre).

Resolución de 18 de septiembre de 2017, de la Dirección General de Ayudas Directas y de Mercados, por la que se modifica la de de 25 de julio de 2017, por la que se aprueba y se da publicidad a las instrucciones dictadas para la autorización y validación de las medidas excepcionales de gestión de crisis establecidas por el Reglamento Delegado (UE) 2017/1165 de la Comisión, de 20 de abril de 2017, que establece, con carácter temporal, nuevas medidas excepcionales de ayuda a los productores de determinadas frutas, en el ámbito de la Comunidad Autónoma de Andalucía (BOJA 184, de 25 de septiembre).

Orden de 19 de septiembre de 2017, por la que se aprueba el Reglamento Específico de Producción Integrada de frutos rojos: Arándanos y Frambuesa y Mora (BOJA 184, de 25 de septiembre).

Resolución de 3 de octubre de 2017, de la Secretaría General de Fondos Europeos Agrarios, por la que se adoptan los porcentajes de reducción y exclusiones de los pagos en el Marco de la Política Agrícola Común por incumplimientos de requisitos y normas en materia de condicionalidad, para la campaña 2017 (BOJA 198, de 16 de octubre).

Orden de 16 de octubre de 2017, por la que se crea la Oficina Comarcal Agraria de Los Pedroches III, se redefine el ámbito territorial de la Oficina Comarcal Agraria de Los Pedroches I y se modifica la Orden de 23 de febrero de 1999, que se cita (BOJA 201, de 19 de octubre). 
Resolución de 19 de octubre de 2017, de la Dirección General de Ayudas Directas y de Mercados, por la que se acuerda la modificación del plazo concedido a los agricultores para la presentación de Comunicaciones de Cesión de Derechos de Pago Básico de la Campaña 2018, en el ámbito de la Comunidad Autónoma de Andalucía (BOJA 207, de 27 de octubre).

Acuerdo de 14 de noviembre de 2017, del Consejo de Gobierno, por el que se aprueba la formulación de la Estrategia Andaluza para la Competitividad de la Industria Transformadora de los Productos de la Pesca y la Acuicultura (BOJA 221, de 14 de noviembre).

Orden de 23 de noviembre de 2017, por la que se adaptan las jornadas y los horarios de las actividades de marisqueo y pesca profesional en el litoral andaluz (BOJA 228, de 28 de noviembre; corrección de errores en el BOJA 239, de 15 de diciembre).

Resolución de 28 de noviembre de 2017, de la Dirección General de Ayudas Directas y de Mercados, por la que se hacen públicos los criterios para la aplicación de las reducciones, sanciones y exclusiones en caso de incumplimiento de los requisitos y condiciones de admisibilidad, compromisos y otros requisitos obligatorios, en relación con las operaciones de la Medida 10: Agroambiente y Clima, de la Medida 11: Agricultura Ecológica y de la Medida 13: Pago a zonas con limitaciones naturales u otras limitaciones específicas, incluida en el Programa de Desarrollo Rural de Andalucía 2014-2020, gestionadas por la Dirección General de Ayudas Directas y de Mercados (BOJA 235, de 11 de diciembre).

Resolución de 30 de noviembre de 2017, de la Dirección General de la Producción Agrícola y Ganadera, por la que se publica la Instrucción sobre modificaciones del periodo de conversión en producción ecológica (RRPG, APG, RPG) (BOJA 238, de 14 de diciembre).

Resolución de 4 de diciembre de 2017, de la Dirección General de la Producción Agrícola y Ganadera, por la que se establecen nuevas zonas demarcadas de los organismos nocivos Epitrix papa y Epitrix cucumeris, así como medidas fitosanitarias obligatorias para su control, en la provincia de Cádiz (BOJA 236, de 12 de diciembre).

Orden de 11 de diciembre de 2017, por la que se modifica la Orden de 19 de junio de 2017, por la que se regula el procedimiento de selección de las estrategias de desarrollo local participativo en las zonas pesqueras de Andalucía para el período 2014-2020 y de reconocimiento de los grupos de acción locales del sector pesquero, y se establecen las bases reguladoras para la concesión de las ayudas a proyectos acogidos a dichas estrategias (BOJA 238, de 14 de diciembre). 
Acuerdo de 12 de diciembre de 2017, del Consejo de Gobierno, por el que se aprueba el Plan Estratégico para la Agroindustria de Andalucía, horizonte 2020 (BOJA 241, de 19 de diciembre).

Corrección de errata de la Resolución de 23 de agosto de 2017, de la Dirección General de la Producción Agrícola y Ganadera, por la que se actualizan las sustancias activas fitosanitarias incluidas en el control integrado de los Reglamentos Específicos de Producción Integrada de Ajo, Alfalfa, Algodón, Almendro, Arroz, Cítricos, Espárragos, Flor cortada, Fresa, Frutales de Hueso, Frutales de Pepita, Cultivos Hortícolas Protegidos, Lechuga al aire libre, Olivar, Patata, Remolacha azucarera, Tomate para transformación industrial, Vid (uva para vinificación) y Zanahoria y Chirivía, BOJA 167, de 31 de agosto (BOJA 170, de 5 de septiembre).

Corrección de errores del Decreto 70/2016, de 1 de marzo, por el que se establece la organización y el régimen de funcionamiento del organismo pagador de los gastos financiados por el Fondo Europeo Agrícola de Garantía y por el Fondo Europeo Agrícola de Desarrollo Rural en la Comunidad Autónoma de Andalucía y se designa al organismo de certificación, BOJA 43, de 4 de marzo (BOJA 213, de 7 de noviembre).

\section{CONSEJERÍA DE MEDIO AMBIENTE Y ORDENAGIÓN DEL TERRITORIO}

Decreto 143/2017, de 29 de agosto, por el que se acuerda la formulación del Plan de Ordenación del Territorio de la Costa del Sol Occidental de la provincia de Málaga (BOJA 174, de 11 de septiembre).

Resolución de 7 de septiembre de 2017, de la Delegación Territorial de Medio Ambiente y Ordenación del Territorio en Almería, sobre recogida de hongos en los terrenos forestales de la provincia (BOJA 185, de 26 de septiembre).

Decreto 150/2017, de 19 de septiembre, por el que se aprueba el Plan de Ordenación de los Recursos Naturales del ámbito Los Alcornocales, se amplía el ámbito territorial del Parque Natural Los Alcornocales y de la Zona de Especial Protección para las Aves Los Alcornocales (ES0000049), y se aprueba el Plan Rector de Uso y Gestión del Parque Natural Los Alcornocales (BOJA 194, de 9 de octubre).

Resolución de 21 de septiembre de 2017, de la Dirección General de Planificación y Gestión del Dominio Público Hidráulico, por la que se hace pública la Resolución de 25 de julio de 2017, de la Dirección General de Planificación y Gestión del Dominio Público Hidráulico, por la que se adoptan las medidas dispuestas en el artículo 54 de la Ley de Aguas de Andalucía, para mejorar el estado cuantitativo y 
cualitativo de la masa de agua subterránea 060.013 Campo de Dalías-Sierra de Gádor, Almería (BOJA 193, de 9 de octubre).

Orden de 11 de octubre de 2017, por la que se amplía para el año 2017 la época de peligro alto de incendios forestales (BOJA 197, de 13 de octubre).

Resolución de 27 de octubre de 2017, de la Delegación Territorial de Medio Ambiente y Ordenación del Territorio en Jaén, sobre recogida de setas en los terrenos forestales de la provincia (BOJA 211, de 3 de noviembre).

Acuerdo de 7 de noviembre de 2017, del Consejo de Gobierno, por el que se aprueba el Plan de Recuperación y Conservación de Invertebrados Amenazados y Fanerógamas del Medio Marino (BOJA 219, de 15 de noviembre).

Resolución de 7 de noviembre de 2017, de la Delegación Territorial de Medio Ambiente y Ordenación del Territorio en Granada, sobre recogida de setas en los terrenos forestales de la provincia (BOJA 221, de 17 de noviembre).

Decreto 191/2017, de 28 de noviembre, por el que se declara la zona especial de conservación Sierras de Cazorla, Segura y Las Villas (ES0000035) y se aprueban el Plan de Ordenación de los Recursos Naturales y el Plan Rector de Uso y Gestión del Parque Natural Sierras de Cazorla, Segura y Las Villas (BOJA 246, de 27 de diciembre).

Orden de 29 de noviembre de 2017, por la que se aprueba el Reglamento de organización y funcionamiento del Consejo Andaluz de Ordenación del Territorio y Urbanismo (BOJA 233, de 5 de diciembre).

Resolución de 19 de diciembre de 2017, de la Secretaría General de Medio Ambiente y Cambio Climático, por la que se determinan las excepciones a la recuperación de costes de los cánones de regulación y tarifas de utilización del agua en las Cuencas Intracomunitarias de la Comunidad Autónoma de Andalucía para el ejercicio 2017 (BOJA 246, de 27 de diciembre).

Resolución de 19 de diciembre de 2017, de la Dirección General de Infraestructuras y Explotación del Agua, por la que se prorroga el canon de trasvase Guadiaro-Majaceite para el ejercicio 2017 (BOJA 246, de 27 de diciembre).

Resolución de 19 de diciembre de 2017, de la Dirección General de Infraestructuras y Explotación del Agua, por la que se prorrogan los cánones de regulación y tarifas de utilización del agua vigentes en las cuencas intracomunitarias de la Comunidad Autónoma de Andalucía para los ejercicios 2017 y 2018 (BOJA 247, de 28 de diciembre). 
Resolución de 23 de diciembre de 2017, de la Dirección General de Infraestructuras y Explotación del Agua, por la que se aprueba el canon de trasvase Guadiaro-Majaceite, ejercicio 2018 (BOJA 248, de 29 de diciembre). 